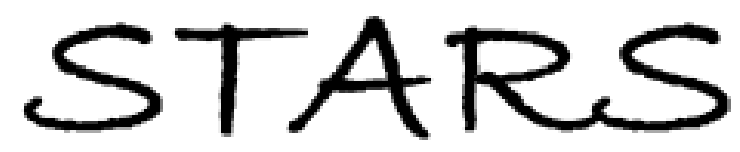

University of Central Florida

STARS

$1-1-2010$

\title{
The 1998 November 14 Occultation of Gsc 0622-00345 By Saturn. li. Stratospheric Thermal Profile, Power Spectrum, And Gravity Waves
}

Joseph Harrington

University of Central Florida

Richard G. French

Katia Matcheva

Find similar works at: https://stars.library.ucf.edu/facultybib2010

University of Central Florida Libraries http://library.ucf.edu

This Article is brought to you for free and open access by the Faculty Bibliography at STARS. It has been accepted for inclusion in Faculty Bibliography 2010s by an authorized administrator of STARS. For more information, please contact STARS@ucf.edu.

\section{Recommended Citation}

Harrington, Joseph; French, Richard G.; and Matcheva, Katia, "The 1998 November 14 Occultation of Gsc 0622-00345 By Saturn. Ii. Stratospheric Thermal Profile, Power Spectrum, And Gravity Waves" (2010).

Faculty Bibliography 2010s. 230.

https://stars.library.ucf.edu/facultybib2010/230

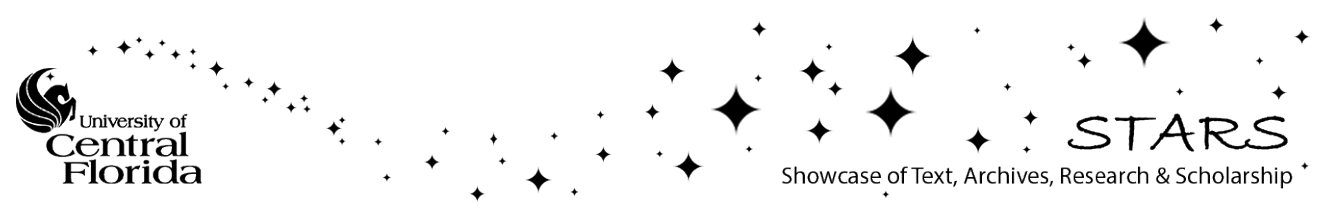




\title{
THE 1998 NOVEMBER 14 OCCULTATION OF GSC 0622-00345 BY SATURN. II. STRATOSPHERIC THERMAL PROFILE, POWER SPECTRUM, AND GRAVITY WAVES
}

\author{
Joseph Harrington ${ }^{1,4}$, Richard G. French $^{2,4}$, and Katia Matcheva ${ }^{3}$ \\ ${ }^{1}$ Planetary Sciences Group, Department of Physics, University of Central Florida, Orlando, FL 32816-2385, USA; jh@physics.ucf.edu \\ ${ }^{2}$ Astronomy Department, Wellesley College, Wellesley, MA 02481, USA; rfrench@wellesley.edu \\ ${ }^{3}$ Department of Physics, University of Florida, P.O. Box 118440, Gainesville, FL 32611, USA; katia@ phys.ufl.edu \\ Received 2009 July 23; accepted 2010 April 7; published 2010 May 19
}

\begin{abstract}
On 1998 November 14, Saturn and its rings occulted the star GSC 0622-00345. The occultation latitude was $55.5 \mathrm{~S}$. This paper analyzes the $2.3 \mu \mathrm{m}$ light curve derived by Harrington \& French. A fixed-baseline isothermal fit to the light curve has a temperature of $140 \pm 3 \mathrm{~K}$, assuming a mean molecular mass of 2.35 AMU. The thermal profile obtained by numerical inversion is valid between 1 and $60 \mu$ bar. The vertical temperature gradient is $>0.2 \mathrm{~K} \mathrm{~km}^{-1}$ more stable than the adiabatic lapse rate, but it still shows the alternating-rounded-spiked features seen in many temperature gradient profiles from other atmospheric occultations and usually attributed to breaking gravity (buoyancy) waves. We conduct a wavelet analysis of the thermal profile, and show that, even with our low level of noise, scintillation due to turbulence in Earth's atmosphere can produce large temperature swings in light-curve inversions. Spurious periodic features in the "reliable" region of a wavelet amplitude spectrum can exceed $0.3 \mathrm{~K}$ in our data. We also show that gravity-wave model fits to noisy isothermal light curves can lead to convincing wave "detections." We provide new significance tests for localized wavelet amplitudes, wave model fits, and global power spectra of inverted occultation light curves by assessing the effects of pre- and post-occultation noise on these parameters. Based on these tests, we detect several significant ridges and isolated peaks in wavelet amplitude, to which we fit a gravity wave model. We also strongly detect the global power spectrum of thermal fluctuations in Saturn's atmosphere, which resembles the "universal" (modified Desaubies) curve associated with saturated spectra of propagating gravity waves on Earth and Jupiter.
\end{abstract}

Key words: atmospheric effects - methods: statistical - occultations - planets and satellites: atmospheres - planets and satellites: individual (Saturn) - waves

\section{INTRODUCTION}

Earth-based occultations remain an attractive method for measuring the thermal profile in the $1-100 \mu$ bar region of a planetary atmosphere. Many such profiles for Saturn were recorded during the 28 Sgr occultation of 1989 July 3, which sampled the equatorial region from $6.6 \mathrm{~N}-15.2 \mathrm{~S}$ latitude (Hubbard et al. 1997). There is a single profile for the north polar region (Cooray et al. 1998; 82.5-85 $\mathrm{N}$ ), and a northern low-latitude profile from the same event (French et al. 1999; $19.16 \mathrm{~N})$. Saturn's central flash probes much deeper, around 2.5 mbar; Nicholson et al. (1995) obtained IR images of the flash during the $28 \mathrm{Sgr}$ event, from which they inferred the zonal wind profile of the sampled latitudes along Saturn's limb.

Occultations observed by a spacecraft near a giant planet probe the troposphere from the cloud deck ( $\sim 1$ bar) to the mbar level at radio and infrared wavelengths. They probe the upper stratosphere and thermosphere $(<1 \mu$ bar $)$ in the ultraviolet. Earth-based visual and infrared occultations measure the thermal structure of the intervening mesosphere and stratosphere regions, which are not well sampled by spacecraft experiments. For Saturn, the Pioneer radio (Kliore et al. 1980; Lindal et al. 1985) and Voyager 2 extreme ultraviolet solar and stellar observations (Smith et al. 1983) sensed the equatorial region only. The Voyager 1 radio occultation sensed $75^{\circ} \mathrm{S}$ (Tyler et al. 1981), while the Voyager 2 radio occultations sensed $36^{\circ} .5 \mathrm{~N}$ and $31^{\circ} \mathrm{S}$ (Tyler et al. 1982). The Cassini radio experiment has performed

\footnotetext{
4 Visiting Astronomer at the Infrared Telescope Facility, which is operated by the University of Hawaii under Cooperative Agreement No. NCC 5-538 with the National Aeronautics and Space Administration, Science Mission Directorate, Planetary Astronomy Program.
}

a number of radio occultations in the equatorial region as well as at middle and high latitudes of Saturn (Nagy et al. 2006; Kliore et al. 2009). The Cassini Ultraviolet Imaging Spectrograph stellar occultation probed the upper atmosphere at $40^{\circ} \mathrm{S}$ and $66^{\circ} \mathrm{N}$ (Shemansky 2008), and the Cassini Composite Infrared Spectrometer mapped Saturn's thermal atmospheric emission, resulting in temperature maps for both hemispheres for pressures ranging from $0.1 \mathrm{mbar}$ to about 700 mbar (Flasar et al. 2005). The Cassini Visual and Infrared Mapping Spectrometer also has the capability of observing spectrally resolved near-infrared stellar occultations by Saturn's atmosphere (Brown et al. 2004).

Temperature profiles for a variety of atmospheres from both occultations and in situ observations show quasi-periodic structures that are usually attributed to propagating waves. Waves have been reported on Venus (Hinson \& Jenkins 1995), Earth (Fritts \& Alexander 2003, and references therein), Mars (Creasey et al. 2006; Fritts et al. 2006), Jupiter (French \& Gierasch 1974; Young et al. 1997, 2005; Raynaud et al. 2003, 2004), Saturn (Cooray et al. 1998; Fouchet et al. 2008), Titan (Sicardy et al. 1999), Uranus (Young et al. 2001), Neptune (Roques et al. 1994), and Pluto (Person et al. 2008; Hubbard et al. 2009; Toigo et al. 2010). Both the behavior of individual waves and the form of wave power spectra can reveal properties of the underlying atmosphere. For example, the forcing, propagation, and dissipation of the waves both contribute to and depend on the sources and sinks of energy in the atmosphere, the background thermal state, and eddy and molecular diffusion.

On 1998 November 14, Saturn and its rings occulted GSC 0622-00345, as predicted by Bosh \& McDonald (1992). We obtained a light curve for atmospheric immersion, based on infrared imaging observations at the NASA Infrared Telescope 
Table 1

Isothermal Fit Results

\begin{tabular}{lcc}
\hline \hline \multicolumn{1}{c}{ Parameter } & Baselines Free & Baselines Fixed \\
\hline Half-light time (UTC) & $11: 18: 47.14 \pm 0.18$ & $11: 18: 46.59 \pm 0.17$ \\
Full flux & $1.0157 \pm 0.0028$ & 1 (not fit) \\
Background & $-0.0150 \pm 0.0013$ & 0 (not fit) \\
Scale height, $H(\mathrm{~km})$ & $50.7 \pm 1.1$ & $44.7 \pm 0.9$ \\
Temperature, $T(\mathrm{~K})$ & $159 \pm 4$ & $140 \pm 3$ \\
\hline
\end{tabular}

Facility (IRTF) on Mauna Kea, HI. The high signal-to-noise ratio $(\mathrm{S} / \mathrm{N})$ allowed us to determine the vertical temperature profile of Saturn's stratosphere at $55^{\circ} .5 \mathrm{~S}$ latitude, a region not sampled by previous stellar occultation observations (see Figure 1 and Table 1 of Harrington \& French 2010, hereafter Paper I).

Paper I presents the light curve and describes the new methods used to acquire and derive it. This paper presents the scientific analysis of the light curve. Subsequent sections cover isothermal model fits, numerical inversions to derive the thermal profile, noise tests, local and global wavelet spectrum analysis, a gravitywave model based on wavelet reconstruction, exploration of the "universal" power spectrum of gravity waves, discussion of the global power spectrum, and our conclusions. For each analysis, we present new significance tests that determine the effects of real (non-Gaussian) noise.

\section{ISOTHERMAL FITS}

Table 1 presents the results of isothermal model fits to the light curve with free and fixed baselines. The free baselines flank the calculated values by over $1.5 \%$ of full flux, a huge deviation given the high accuracy of the baseline determination. The second fit fixes the baselines at their calculated values. For the latter case, the derived scale height is very close to that in the good region of the inversion presented below.

Isothermal fits can give approximate light-curve parameters that do not depend on many assumptions and that are unique. However, the vastly different values for temperature, $T$, and scale height, $H$, between the two cases and the poorly fit baselines in the free-baselines case indicate that isothermal models do not approximate this atmosphere well. Both models have long, nonzero tails that stand well above the data (see Figure 2 of Paper I). The light curve also contains many spikes with amplitudes that are many times the noise level. The spikes and the low-valued tail are features of the observations that are not in the model but that strongly influence where the fit falls. Introducing a temperature gradient to the model might improve the fit, but that does not address the numerous spikes. Inversion is thus the proper analytic approach.

Both here and in the inversion that follows, we use the mean molecular mass to convert $H$ to $T$. The $\mathrm{He} / \mathrm{H}_{2}$ volume mixing ratio for Saturn determined by Conrath \& Gautier (2000) is in the range $0.11-0.16$. We adopt a value of 0.135 and a $\mathrm{CH}_{4}$ mixing ratio of $(4.7 \pm 0.2) \times 10^{-3}$ (Fletcher et al. 2009), resulting in a mean molecular mass of 2.35 AMU. The uncertainty in $\mathrm{He} / \mathrm{H}_{2}$ dwarfs the uncertainty in any other constituent. The value of 2.135 AMU used by Hubbard et al. (1997) and many prior workers is much smaller. One must be careful to adjust temperatures and adiabatic lapse rates to the same mean molecular mass when making comparisons.

\section{TEMPERATURE PROFILE}

We applied an Abel transform to the normalized light curve under the usual assumptions that the atmosphere is radially symmetric and that ray crossing is not substantial (French et al. 1978; in grazing occultations, unlike ours, these assumptions may be violated, see Cooray \& Elliot 2003). This produced $T$, pressure $(P)$, and number density $(n)$ as a function of height above the half-light level $(z)$. Table 2 and Figure 1 show our parameters and results. We rebinned the light curve prior to the inversion into time intervals corresponding to vertical atmospheric layers of equal thickness $(1 \mathrm{~km})$. This is high enough resolution to preserve the intensity spikes in the light curve and is roughly comparable to the $1.2 \mathrm{~km}$ Fresnel scale (Paper I). Saturn is very oblate, so at any point on the surface the radius of curvature depends on both the latitude and the direction being considered (e.g., north-south versus east-west). Using the radius of curvature along the line of sight at the half-light latitude accounts for planet's oblateness. The derived vertical refractivity profile is proportional to the density profile. The $T$ and $P$ profiles then result from integrating the hydrostatic equation and applying the ideal gas law (French et al. 1978).

The inversion process determines the unique vertical temperature profile such that a forward model of the occultation would reproduce the observed light curve exactly, subject to assumptions just mentioned. Unfortunately, the inversion cannot distinguish between noise and atmosphere-induced variations in stellar intensity. Both random and systematic noise in the observations can thus seriously affect the derived temperature profile (see Elliot et al. 2003 for an extensive review, and references cited therein). Initially, the photometric noise in the upper baseline completely overwhelms the actual refractive effects of the tenuous upper atmosphere. The noise introduces $\sim 100 \mathrm{~K}$ swings in the upper part of the derived thermal profile, and these unphysical swings bias the results at the onset of the inversion. In deeper layers, the uncertainties associated with this initial condition are less significant. Eventually, refractive defocussing of the starlight is so large that the noise in the lower baseline of the light curve dominates the faint signal. This results in unphysical temperature variations and trends at the deepest atmospheric

Table 2

Inversion Parameters and Results

\begin{tabular}{lcc}
\hline \hline \multicolumn{1}{c}{ Description } & Value & Comment \\
\hline Gravity at half-light, $g$ & $11.06 \mathrm{~m} \mathrm{~s}^{-2}$ & \\
Refractivity at STP & $1.24 \times 10^{-4}$ & \\
Mean molecular mass & $2.35 \mathrm{AMU}$ & Conrath \& Gautier (2000) \\
Local rad. of curv. & $64,307.9 \mathrm{~km}$ & Along line of sight \\
$T / H$ & $3.126 \mathrm{~K} \mathrm{~km}^{-1}$ & \\
Adiabatic lapse rate, $\Gamma$ & $-0.992 \mathrm{~K} \mathrm{~km}^{-1}$ & \\
\hline
\end{tabular}

Note. ${ }^{\text {a }}$ Standard temperature and pressure. 


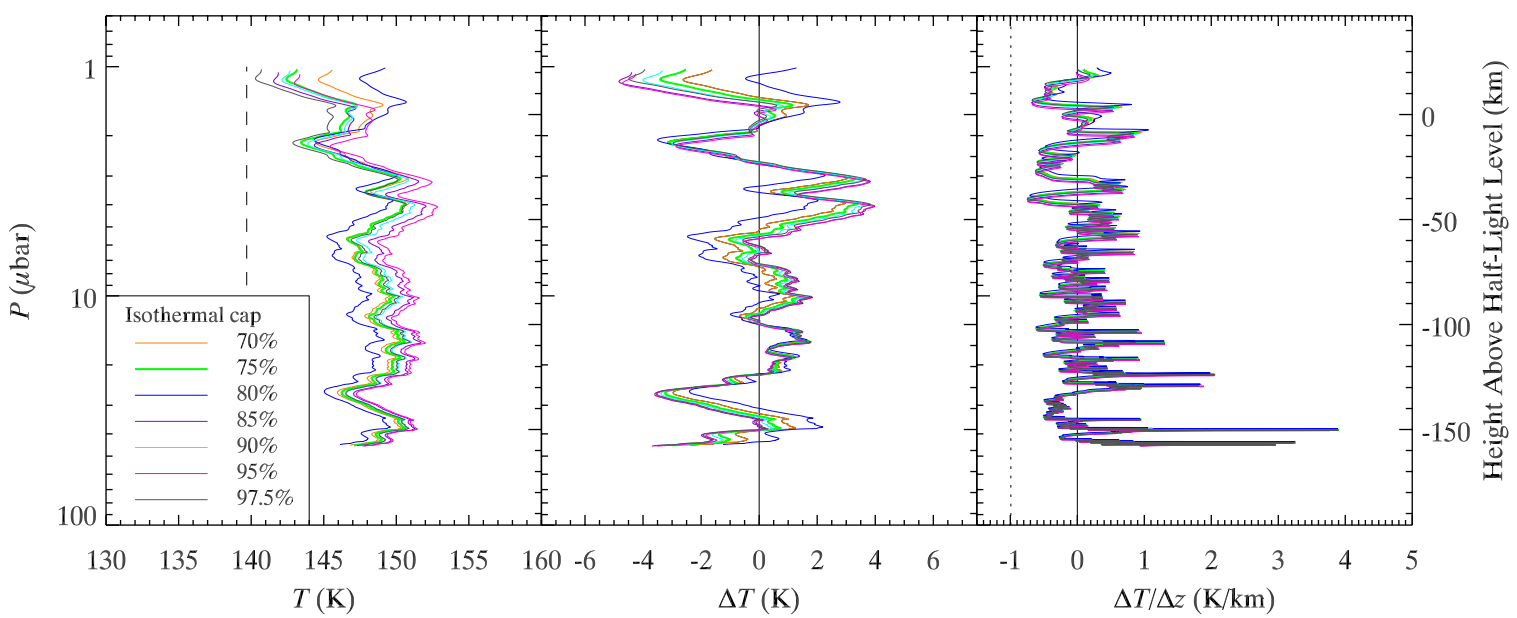

Figure 1. Family of thermal profiles derived by numerical inversion. The light curve inverted for each curve has an isothermal cap that ends at the flux level indicated in the key. We used the 75\% curve (green line in the middle of the group) for subsequent analyses. The data are plotted vs. pressure (left axis), with the approximate corresponding altitude scale given on the right axis. Left: temperature profiles. The dashed line shows the temperature of our isothermal fit. Middle: the same data after removal of a linear fit to each profile. This shows that the shape and amplitude of the small-scale temperature fluctuations is consistent among the profiles, that there are very small differences in their altitudes, and that the deviations from a linear thermal profile are no more than about $\pm 4 \mathrm{~K}$. Right: vertical temperature gradient vs. altitude, derived from the left panel's data. The atmosphere is statically stable over this altitude range, since the temperature gradient is separated from the adiabatic lapse rate (dashed line). Nonetheless, we still see alternating rounded and spiked structures, which are seen in many other profiles where the vertical temperature gradient approaches adiabatic.

layers probed during the occultation (see, e.g., Raynaud et al. 2004). As a rough guide, French et al. (1978) showed that, for high-quality, Earth-based stellar occultations, the valid region of the derived temperature profile ranges from about 0.5 to -3.5 $H$ above and below the half-light level.

Several strategies have been adopted to minimize the effects of the unstable initial condition of the inversion process. Elliot \& Young (1992) and Elliot et al. (2003) explored a range of models that fit the upper part of the light curve assuming a power-law dependence of temperature with radius. This is an extension of the strategy developed by French et al. (1978) of replacing the upper part of the light curve by the best isothermal fit to that restricted part of the data. In the absence of detailed knowledge of the upper stratospheric temperature structure above the occultation region, we adopt the minimalist assumption that the atmospheric region sounded by the upper part of the light curve is isothermal. Effectively, we assume that the upper atmosphere above the inversion's reliable region does not have large-scale temperature fluctuations and is comparable in mean temperature to the valid region. A comparison of $28 \mathrm{Sgr}$ and Voyager UV stellar occultation observations bears this out for Saturn (Hubbard et al. 1997). The stability of the hybridlight-curve inversion depends on the length of this isothermal "cap." If the cap extends only from the upper baseline to the $99 \%$ level (in units of normalized stellar flux), then the noise in the subsequent upper part of the observed light curve will still produce spurious temperature variations at the onset of the inversion. As the cap length increases, the inversion stabilizes, eventually contaminating the inversion's valid region. The optimal cap is large enough to give a stable inversion but ends above the valid region.

The left panel of Figure 1 shows the thermal structure derived from a suite of light-curve inversions with seven different isothermal caps ending at $97.5 \%-70 \%$ of the full stellar signal (we computed additional caps outside this range). The order of the curves reflects the sensitivity of the inversion to noise and atmospheric structure that immediately follow the isothermal cap. The degree of uncertainty in the inversion is shown by the spread between the profiles. We have used the $75 \%$ cap in all subsequent analyses. Elliot et al. (2003) show that a 50\% cap results in about a 3\% error in the derived temperature at the onset of the underlying inversion region, for an isothermal light curve with S/N per scale height of 200 in the presence of white noise. Since our goal is to investigate vertical variations in temperature as well as to estimate the mean temperature, we have adopted a compromise of replacing only the upper $25 \%$ of the light curve by an isothermal model.

The temperature variations are more clearly seen in the middle panel of Figure 1, which shows the deviations of each of the profiles from a linear fit to that profile. The right panel shows the vertical temperature gradient for each inversion. The vertical dashed line corresponds to the adiabatic lapse rate. The atmosphere is locally stable against convection from 1-60 $\mu$ bar. Some occultation temperature gradient profiles (e.g., Figure 10 of Raynaud et al. 2003, Figure 7 of Raynaud et al. 2004, and others cited therein) have oscillations with a rounded shape on the low $\Delta T / \Delta z$ side and a narrow, spiked shape on the high side, and so does ours, particularly at depth. The asymmetric rounding has been attributed to gravity wave breaking as the profile's gradient approaches the adiabatic lapse rate. Saturn's adiabatic lapse rate is separated from the negative-side extrema in our profile's gradient by $0.2 \mathrm{~K} \mathrm{~km}^{-1}$ everywhere, and generally by much larger amounts. One might expect that, as waves propagate vertically and increase in amplitude by virtue of energy conservation, they will eventually become superadiabatic and result in wave breaking, but this is not always reflected in the retrieved vertical temperature profiles. Similar results to ours have been found for Pluto (see Figure 4 of Young et al. 2008). The occultation profile integrates over a large atmospheric path length, so there may be local gradient instabilities not seen in occultation inversions.

\section{NOISE TESTS}

It is not a simple matter to quantify in detail the effect of lightcurve noise on the numerical inversions. The inferred structure at a given atmospheric level is contaminated by errors in the derived refractivity of all overlying levels. French et al. (1978) showed that the correlation length scale of the inversion process extends to well over a scale height above any given pressure 
Table 3

Inversion Set Temperature Ranges and Power-law Fits to Global Wavelet Spectra

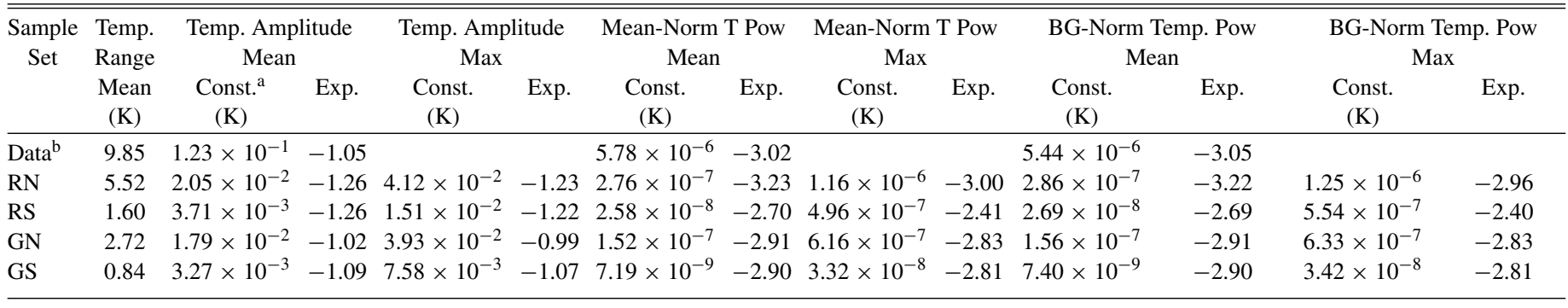

Notes.

${ }^{\text {a }}$ Const. and Exp. refer to the constant and exponent (power) of the power-law fits (Section 7).

${ }^{b}$ Range is average range for different caps. Power law is for $75 \%$ cap.

level, as dictated by the width of the kernel in the integral equation for the Abel transform. Thus, even uncorrelated white noise in the light curve results in correlated errors in the derived thermal profile.

The problem is even more complex if the input light-curve noise is correlated, and almost any baseline drift on timescales longer than the exposure time will introduce significant correlation. Causes of such drifts include turbulence and waves in Earth's atmosphere ("seeing"), atmospheric transparency variations, and pointing drifts. The latter can be problematic in spacecraft instruments with few spatial channels (e.g., few pixels) or with pointing-dependent sensitivity.

Although previous investigators have explored the consequences of white noise to the mean temperature determined by inversion (e.g., French et al. 1978; Elliot et al. 2003), there has been no systematic study of the effects of noise on wave analyses, and little consideration of correlated noise. To assess the significance level of features in our derived profiles and subsequent analyses, we added both Gaussian and real noise to an isothermal model light curve. We created 25 realizations of Gaussian noise with the same standard deviation as our upper baseline. To eliminate the baseline uncertainty issue, we added this noise only below the $75 \%$ light level. For the real noise tests, we took a section of our upper baseline, removed a loworder polynomial, and repeated the section several times. The polynomial ensures that the sections have zero mean and meet without a discontinuity. We shifted the resulting data vector by three different amounts so that specific noise spikes would appear at three different locations along the synthetic light curve. We added this to the isothermal curve starting at each of the cap levels to create 21 sets (seven caps times three shifts).

Sources of scintillation noise include both the star and the residual from template subtraction (Paper I). To account for the decrease in the stellar contribution to scintillation, we created two additional sample sets, identical to those above but with the noise scaled by the normalized intensity of the noise-free isothermal model light curve. Most ground-based occultation data sets have similar noise levels on both baselines, indicating that the dominant noise source is residual planetary light and that the unscaled noise analysis is most appropriate for that case. However, reductions in the residual planetary signal are possible in the future, in which case the scaled noise analysis would apply. We label the four sets of noisy isothermal profiles "RN" (real, normal), "RS" (real, scaled), "GN" (Gaussian, normal), and "GS" (Gaussian, scaled).

We inverted all of these light curves and calculated the temperature ranges in the valid regions of each resulting profile. The first columns of Table 3 present the average of all the temperature ranges in each sample set and in the observations. Both types of noise induced large oscillatory structures into profiles that, without noise, should have been straight vertical lines, but real noise had a dramatically larger effect than Gaussian noise. Temperature ranges were typically $2-3 \mathrm{~K}$ for the GN set, although a single outlier had a $6.4 \mathrm{~K}$ range. Within a given shift of the RN set, the profiles and their ranges were mostly similar. Ranges for the three shifts averaged 7.4, 5, and $4.5 \mathrm{~K}$, indicating that the placement of individual light-curve spikes strongly affected the results. The scaled sets had much smaller ranges, but otherwise behaved similarly to their unscaled brethren.

The large-amplitude oscillations in these supposedly isothermal inversions generally have wavelengths of at least a scale height. Real noise is substantially worse than Gaussian noise of the same standard deviation, likely because of its red power spectrum. We assess the power spectrum of the noise and related error and significance issues in Section 7.

\section{WAVELET ANALYSIS}

The observed temperature profiles all show fluctuations with amplitudes as large as $4 \mathrm{~K}$. Small-scale, quasi-periodic structures in atmospheric profiles are often interpreted as inertiagravity waves (French \& Gierasch 1974; Young et al. 1997; Cooray et al. 1998; Raynaud et al. 2003, 2004). To investigate the wave nature of these structures in more detail, we computed the wavelet transform of the valid region. A wavelet transform gives the amplitude or power spectrum as a function of atmospheric depth. Torrence \& Compo (1998) provide a quantitative and accessible wavelet tutorial with software ${ }^{5}$. In a wavelet image of amplitude or power versus wavelength and height, such as we present in Figure 2, a wave train of constant wavelength and amplitude would appear as a constant-brightness, vertical band. If the wavelength varied along the train, the ridge would tilt or curve. Changes in amplitude would appear as varying brightness along the band.

Following Raynaud et al. (2003, 2004), we used the Morlet wavelet with a nondimensional frequency of $6\left(\omega_{0}\right.$ in Equation (1) of Torrence \& Compo 1998). The left panel of Figure 2 shows the valid region of the temperature profile, for which we computed the wavelet amplitudes presented in the right panel. Torrence \& Compo (1998, their Equation (8)) provide a power spectrum "normalization" that allows direct

\footnotetext{
5 http://paos.colorado.edu/research/wavelets/
} 


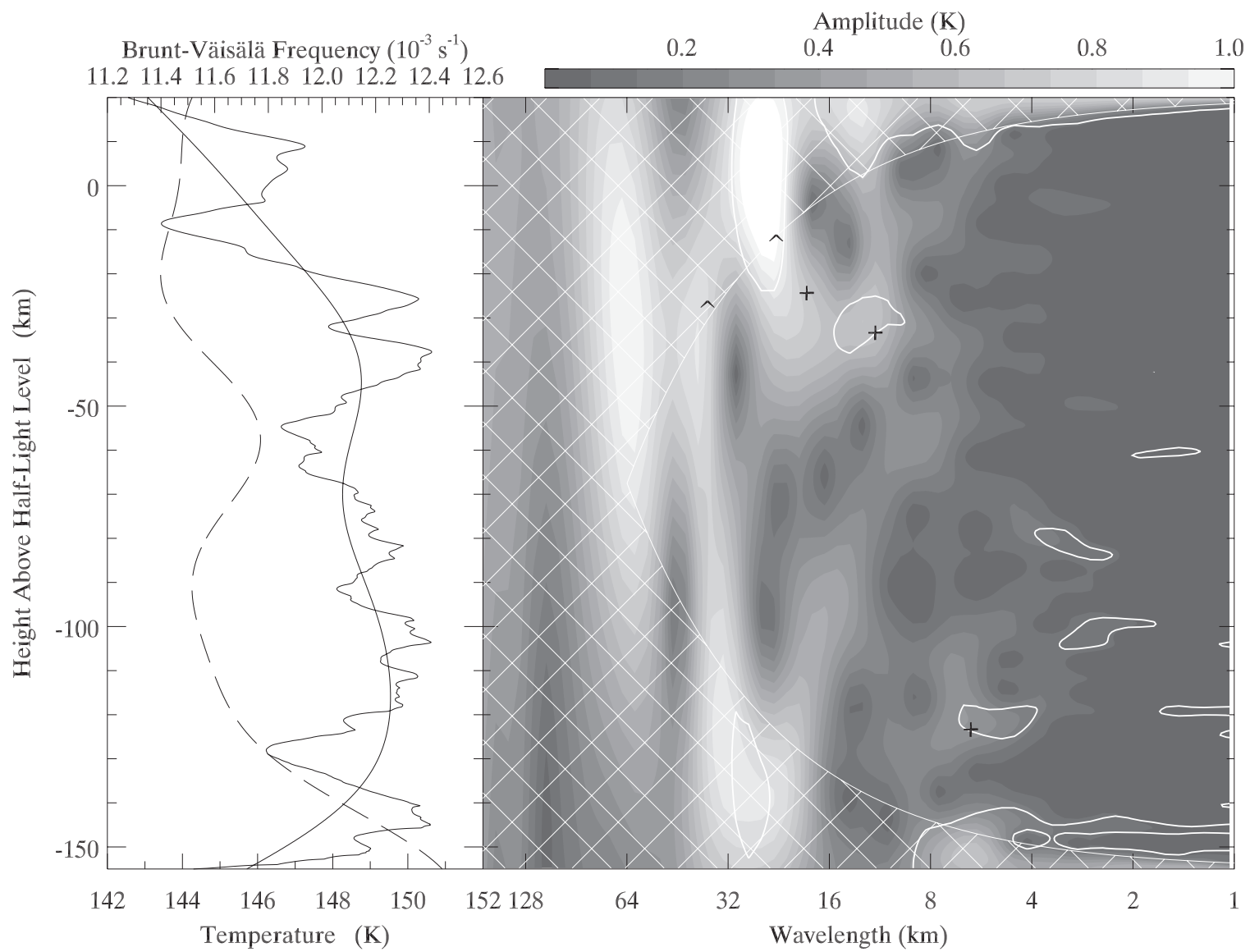

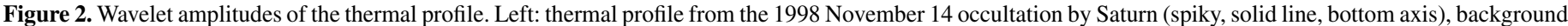

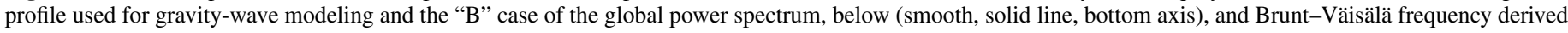

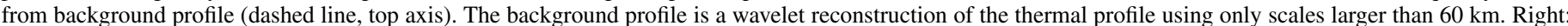

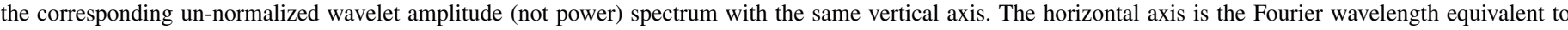

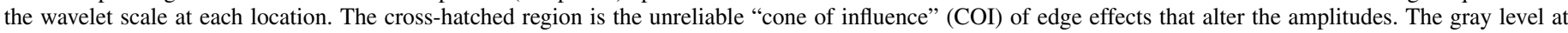

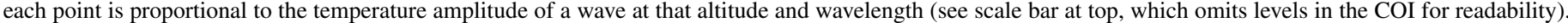

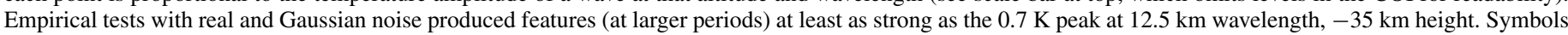

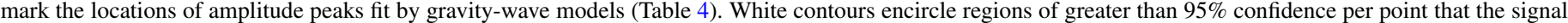
stands significantly above the data's global power spectrum. See Section 5 for discussion, including significance contours.

comparison to Fourier analyses, which we do below. This adjusts for the wavelet spectrum's geometric spacing between frequencies and enables definite integrals of power spectral density (PSD) to compute total power in a wavelength range. Since amplitude spectra are not spectral densities (i.e., one does not integrate them), one must remove the normalization to express amplitudes in Kelvins. We thus keep the adjustment for the power spectra presented herein, but remove it for amplitude spectra. This adjustment and the orthogonal basis set of the transform allow us to recover accurately the amplitudes of synthetic sinusoidal signals inserted into the input data. The cross-hatched region is the so-called cone of influence (COI) of the edges of the data. In this region, points are close enough to the edge of the data that wavelets at those periods extend beyond the data. This effectively averages in zeros from outside the data. Structure within the COI is unreliable, so we ignore it. The profile is dominated by structures with wavelengths longer than $32 \mathrm{~km}$. Few-kilometer, irregular wiggles are superposed, but consistent strength in scales of $5-30 \mathrm{~km}$ is absent. This is reflected in the wavelet transform, where amplitudes drop substantially outside the COI.

We also computed the global wavelet power spectrum (Torrence \& Compo 1998, Equation (22)) by averaging the normalized wavelet power transform over all valid heights that are outside the COI (see Figure 3). This method of computing the power spectrum has advantages over both a single Fourier transform with a window applied to the data and a similar average over a windowed Fourier transform (WFT). The WFT is a local transform similar to a wavelet transform, but its sliding window has a fixed width that affects the computation differently at each wavelength, introducing wavelength-dependent behavior. The wavelet transform's window size scales with the wavelength and thus has the same effect at each wavelength. The local nature of both the WFT and wavelet transform allows one to use the COI to omit points contaminated by edge effects. The result is a dramatic reduction in power spectrum noise, as shown in Figure 3. The new significance test for the global power spectra (see Section 7) could not be meaningfully applied without the superior noise rejection of wavelets.

The data's global spectrum shows a downward-curving trend with superposed peaks. The general trend appears to follow an atmospheric gravity-wave spectrum (see Section 7). The peaks could be discrete gravity waves, which we study with a model (see Section 6). Finally, we compare the global spectra of the data and of our noise tests to establish significance (see Section 7).

Are the features in the global and local wavelet spectra significant? This is really two separate questions: does the 


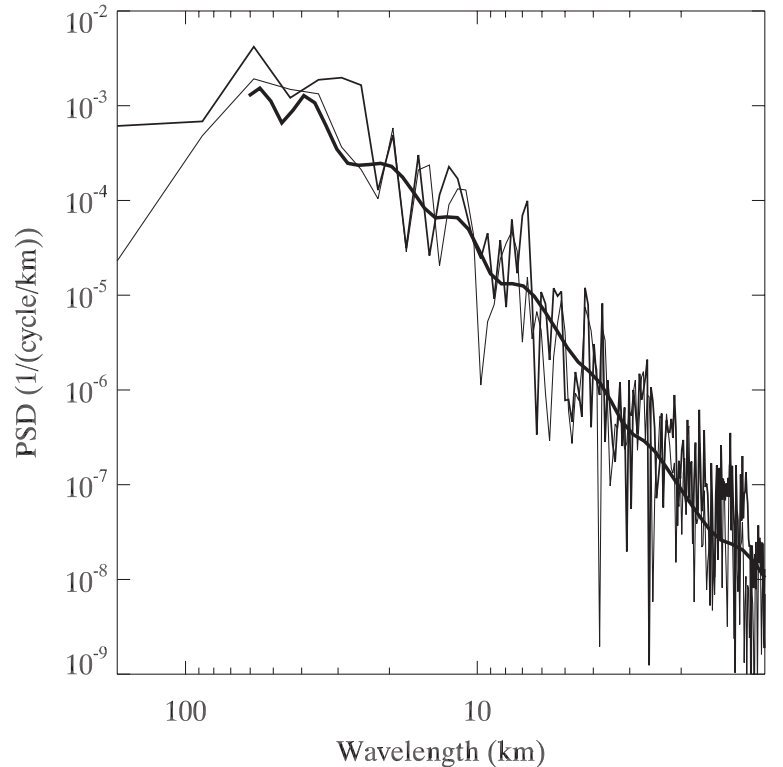

Figure 3. Power spectrum of thermal profile ( $75 \%$ cap, B case of normalized temperature, below) derived in three ways. Thick line: wavelet spectrum averaged outside of COI. Medium line: Fourier transform with Hann window and $8 / 3$ scaling used, e.g., by Young et al. (2005). Thin line: Fourier transform without window or scaling. The wavelet-derived spectrum is much smoother than either Fourier-transform-derived spectrum. The non-Hann Fourier spectrum rises above the other two curves at short wavelength and falls below the Hann version at long wavelength. The high noise level of the Fourier-transformderived spectra would make them unsuitable for use in our significance test.

global spectrum stand significantly above the noise, and do local features stand significantly above the global spectrum? We discuss the latter here and present the former in Section 7.

Torrence \& Compo (1998) developed a rigorous significance test based on a lag-1 autocorrelation noise model, which compares the data to the data shifted by one point, on the assumption of a short correlation length. That test unfortunately does not apply to occultation inversions, since inversions are correlated from any given depth all the way to the top of the atmosphere. Thus, the correlation length varies as much as it possibly can, and is short only at the top of the atmosphere.

We verified that the real and imaginary parts of the power transform each had a Gaussian distribution at a given wavelength. Then, we followed code comments of Torrence \& Compo in applying the $\chi^{2}$ probability distribution to determine the multiplier for the global spectrum that gives the $95 \%$ confidence level (we assume that if the power is significant at a given point, so is the amplitude). In Figure 2, contours encircle regions with power greater than this level. Note that these are not contours of the amplitude transform, since the global power spectrum's normalization varies with wavelength.

The strongest of the significant features that is completely outside the COI has vertical wavelength $\lambda_{z}=12.5 \mathrm{~km}$, a maximum at $z=-35 \mathrm{~km}$, and amplitude $0.7 \mathrm{~K}$ at that level. This feature can be interpreted as a short gravity-wave train, as can several others at shorter wavelengths and much lower amplitudes. It is localized, meaning that it does not extend vertically over the entire data set. One can see, from the left panel in the plot, that this feature lasts at least 1.5 cycles. We show in Figure 4 that it extends over four cycles. Young et al. (2005) also detected several short wave trains in the Galileo Probe data for Jupiter.

Aside from their much-lower amplitude at a given wavelength, the transforms of the noise data sets are qualitatively very similar to that in Figure 2, except that the RS and GS sets have decreasing power at lower altitude (as expected). The large-amplitude (many-K) oscillations in the noise sets generally have wavelengths of at least a scale height, and are in the COI. Wavelet amplitude maxima outside the COI for the GN

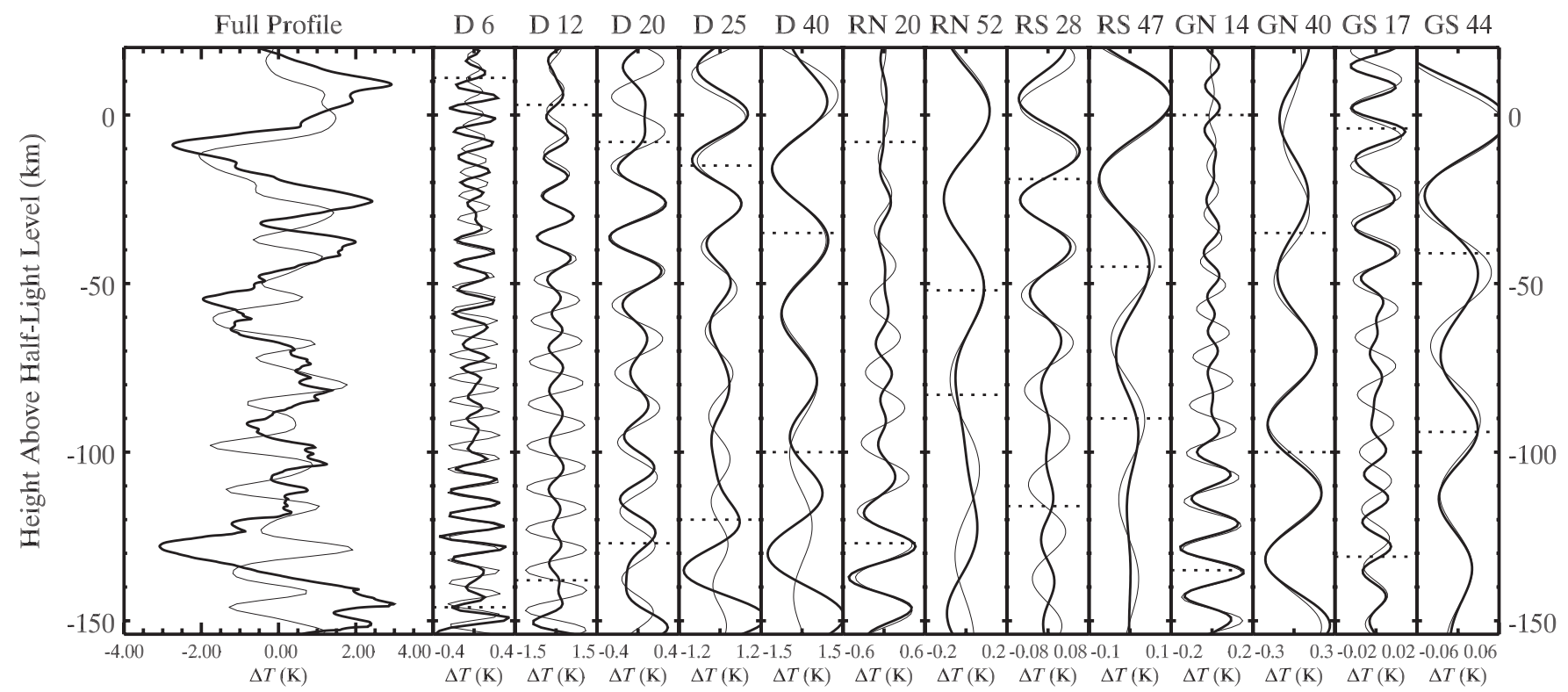

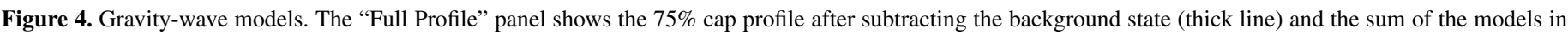

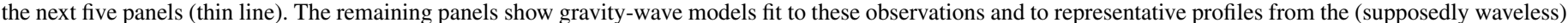

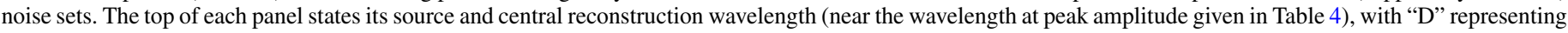

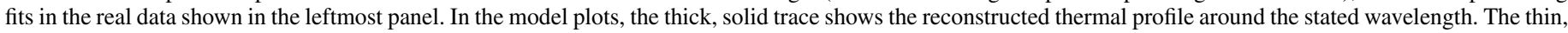

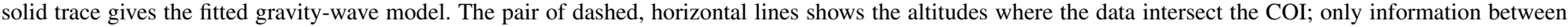

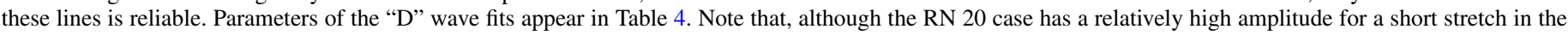

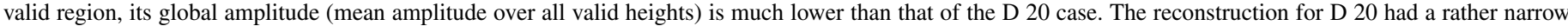

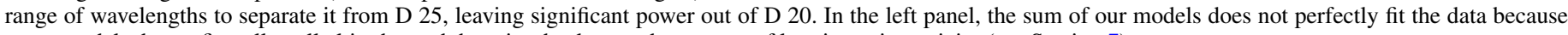
some models do not fit well at all altitudes and there is a background spectrum of low-intensity activity (see Section 7). 
set averaged $\sim 0.13 \mathrm{~K}$, and for the three shifts of the RN set were $0.19,0.30$, and $0.34 \mathrm{~K}$ (these red-noise features appeared at long wavelengths). Maxima are somewhat smaller for the scaled data sets, but this is less meaningful as their maxima now come only from the high-altitude region; given enough samples we would expect similar maxima. In both real and Gaussian cases, about half the tests contained substantial, isolated regions outside the COI with amplitudes that stood above the surrounding features. These regions were broader for the real noise tests, often substantially broader than the ones in Figure 2. The maximum transform amplitude outside the COI often appeared in these features. As we note below, most or all of the data transform outside the COI stands well above the noise. Again, one assesses this best by comparing global spectra. The point here is that one cannot tell signal from noise merely by looking at the pattern presented.

Numerous wave features in the literature have amplitudes smaller than $1 \mathrm{~K}$, often in noisier data sets than this one (e.g., Raynaud et al. 2003, 2004). Such cases likely also have significant, spurious fluctuations in their transforms due to scintillation noise and other terrestrial atmospheric effects, though it is impossible to tell for certain without the test described above, as the degree of such noise is rarely reported and time-correlated noise could conceivably have been better for those observations than that for ours, even if random noise were worse. It thus becomes imperative to apply a wavelengthdependent significance test, such as that presented above. Had we simply used the maximum of our noise transforms, we would have rejected all the encircled regions of Figure 2 as being below $2 \sigma$ significance. By the same token, it becomes difficult to accept such detections without any significance test. There are two classes of features, discrete and global. The significant, discrete features have contours around them in Figure 2. The significant, global features are the bumps in the thick line of Figure 3, which we interpret with a gravity-wave model in the next section and later compare to the noise spectra.

\section{GRAVITY-WAVE MODEL}

The noise transforms do not show the continuous vertical ridges of elevated amplitude one would expect of a wave that propagates undamped through the entire valid region of the temperature profile. However, it may be possible, by fitting an appropriate model, to improve sensitivity to such individual gravity-wave modes in the data, using the vertical coherence that the noise does not exhibit. Raynaud et al. (2003, 2004) fit such models to identify gravity-wave signatures in Jovian temperature profiles derived from two stellar occultations. Note that these two papers use different methods. Raynaud et al. (2003) used three chords from one event to determine both $\lambda_{z}$ and the horizontal wavelength, $\lambda_{h}$. Through their Equation (15) they determine the dissipation level for the wave and point out that the observed $T$ peaks are too high in the atmosphere to be caused by a gravity wave with the derived parameters. In contrast, the event reported by Raynaud et al. (2004) has just a single observation. The horizontal wavelength is a free parameter and fits are consistent with a gravity-wave interpretation.

We follow Raynaud et al. (2004) very closely to test whether waves may be propagating at the wavelengths corresponding to peaks in the global wavelet spectrum shown in Figure 3. These correspond to regions outside the COI and at $\lambda_{z}<60 \mathrm{~km}$ in Figure 2. The first three columns of Table 4 give $\lambda_{z}$, altitude $\left(z_{\max }\right)$, and temperature amplitude $\left(\Delta T\left[z_{\max }\right]\right)$ of five
Table 4

Parameters of the Gravity-wave Fits

\begin{tabular}{lcccccc}
\hline \hline \multicolumn{3}{c}{ Observed Parameters } & & \multicolumn{3}{c}{ Model-dependent Parameters } \\
\cline { 1 - 2 } \cline { 6 - 7 }$\lambda_{z}$ & $z_{\max }$ & $\Delta T\left(z_{\max }\right)$ & & $z_{0}$ & $\lambda_{h}$ & $\begin{array}{c}\text { Period } \\
(\text { minutes })\end{array}$ \\
$(\mathrm{km})$ & $(\mathrm{km})$ & $(\mathrm{K})$ & & $(\mathrm{km})$ & $(\mathrm{km})$ & 13.3 \\
\hline 6.5 & -125 & 0.26 & & -114 & 10 & 13.5 \\
12.5 & -35 & 0.7 & & -143 & 120 & 85.5 \\
20 & -26 & 0.26 & & -10 & 300 & 125.7 \\
25 & $>-15$ & 0.6 & & $>-15$ & 100 & 35.6 \\
40 & $>-30$ & 1 & & $>-30$ & 200 & 42.0 \\
\hline
\end{tabular}

temperature peaks; these are marked in Figure 2. The remaining columns give the altitude of maximum amplitude of the model wave $\left(z_{0}\right), \lambda_{h}$, and the wave period. Physically, $z_{0}$ represents the altitude where wave damping exceeds the natural exponential amplitude growth. The intensity of dissipative processes, $\lambda_{z}$, and $\lambda_{h}$ determine this altitude.

As a wave propagates, variations in the atmosphere's steadystate properties affect its amplitude and vertical wavelength. In a conservative atmosphere, a vertically propagating wave experiences exponential growth of its temperature amplitude as a consequence of the exponential decrease of $n$ and the requirement for energy conservation. If the amplitude becomes large enough for the local temperature gradient to exceed the adiabatic lapse rate, $\Gamma$, the wave becomes unstable and overturns. Dissipative processes such as molecular viscosity, eddy diffusion, thermal conduction, and radiative damping can further limit amplitude growth, causing the wave to deposit its energy in the background atmosphere (Lindzen 1981). Furthermore, variations in the background temperature and vertical shear in the zonal wind can also cause observable changes in the amplitude, vertical wavelength, and phase propagation.

We simulate these effects with a hydrostatic WKB gravitywave model in a rotating atmosphere. The model (Matcheva \& Strobel 1999) includes dissipation by molecular viscosity, eddy diffusion, and thermal conduction. We assume that there is no vertical gradient in the zonal wind. Calculations that included constant wind shear did not improve the fits. We assume scales larger than $60 \mathrm{~km}$ to represent the atmosphere's steady state, although from a single occultation we cannot determine whether an observed structure is transient or static. Our steady-state profile is the inverse wavelet transform of scales larger than $60 \mathrm{~km}$ in the right panel of Figure 2; it appears as the smooth, solid line in the left panel.

The eddy diffusion coefficient, an input in our wave model, parameterizes the intensity of the vertical mixing in the atmosphere. Published values for Saturn's eddy diffusion coefficient differ by more than 2 orders of magnitude (Moses et al. 2000 and references therein). In our model it varies with altitude and is proportional to $n^{-1 / 2}$ (Atreya 1986). We set it to $500 \mathrm{~m}^{-2} \mathrm{~s}$ at the $0.1 \mu$ bar level to agree with Voyager's UVS observations (Smith et al. 1983). The vertical mixing in the atmosphere is important as a wave dissipation mechanism. The degree of wave dissipation is determined by the eddy diffusion coefficient and the wave's vertical and horizontal wavelengths. Since the temperature profile only has vertical information, the horizontal structure is a free parameter. The exact eddy diffusion coefficient value is thus not critical for the wave model because adjusting the horizontal wavelength, $\lambda_{h}$, within a reasonable range can compensate for reasonable changes in the eddy diffusion coefficient without changing the vertical structure.

The wave model parameters are $\lambda_{h}, \lambda_{z}$, temperature amplitude $\Delta T$, and phase $\phi$ at the lower boundary. We adjust the wave 
parameters so that the model fits the observations at $z_{\max }$ (see Table 4). Since the background atmosphere's temperature varies with altitude, so does $\lambda_{z}$ for each wave. We select a short range in $\lambda_{z}$ for each candidate wave, zero all other scales in the wavelet transform, and reconstruct the temperature fluctuations due just to those scales using the inverse transform. We compare the simulated wave and the reconstructed temperature variations within the limits set by the COI. The "D" panels of Figure 4 present the results.

For the purpose of wave identification, one would ideally like to follow the wave signature over several wavelengths and several scale heights, with at least two scale heights below the altitude of wave damping. However, the region outside the COI becomes progressively shorter for larger wavelengths. Therefore, large- $\lambda_{z}$ temperature fluctuations are difficult to interpret uniquely as signatures of propagating waves.

We obtain a relatively good fit for the longest wavelength that we consider $\left(\lambda_{z}=40 \mathrm{~km}\right)$, detecting significant power throughout the observed region. The simulated wave has a $\lambda_{h}=$ $200 \mathrm{~km}$; the corresponding gravity-wave period is 42 minutes. The wave exists below the expected altitude of dissipation (altitude of maximum amplitude) and shows modest amplitude growth. Both the amplitude and the phase of the simulated wave follow the reconstructed temperature fluctuations well. However, despite the apparently good fit, we cannot rule out alternative interpretations, since the reliable part of the reconstruction contains only two wave cycles.

For the rest of the candidates, we have mixed success in fitting the observed temperature fluctuations with a single propagating gravity wave. In general, we are able to fit the reconstructed temperature fluctuations (both amplitude and phase) well over $2-5$ cycles near the peak amplitude, but we are not successful in matching the observations throughout the entire altitude region. Young et al. (2005) also present wave activity in the Galileo Probe data for Jupiter that spans only a few cycles before it disappears. As previously (Raynaud et al. 2004), our requirement for a positive wave identification is much more restrictive, since we require a good fit throughout the profile's valid region.

Our wave model fit to the $25 \mathrm{~km}$ reconstructed scales does a relatively good job following the amplitude of the temperature fluctuations throughout the extent of the data, but it fails to fit the phase of the observations at the bottom of the occultation $(z<-70 \mathrm{~km})$.

In the case of the $20 \mathrm{~km}$ structure, we have a good phase match throughout the sampled region, but the amplitude exhibits a double peak that is difficult to explain with a simple gravitywave model, with or without a constant vertical wind shear. The amplitude fits well within four wavelengths of the top and bottom of the valid region, but overestimates the observed fluctuations in the central part of the profile. A variable wind shear might explain the observed amplitude.

The $12.5 \mathrm{~km}$ structure has a well defined power maximum that is nicely separated from the COI. This and the relatively small vertical scale make the wave analysis more robust. We achieve a good amplitude and phase fit for four wavelengths for $z>-50 \mathrm{~km}$. At $z=-50 \mathrm{~km}$, the amplitude shows a very fast increase with altitude, which is inconsistent with our model. Such a fast amplitude variation can be an indication of significant vertical shear in the zonal wind. If this were the case, however, we should also detect a sudden change in $\lambda_{z}$, but the observed change is not very large.
The temperature reconstruction of the $6.5 \mathrm{~km}$ candidate shows a significant variation in amplitude. These variations can be modeled with some success if one assumes that the background temperature profile retains scales, $L_{z}$, below $60 \mathrm{~km}$ (e.g., $L_{z}>30 \mathrm{~km}$ ). For our background temperature profile $\left(L_{z}>60 \mathrm{~km}\right)$, the model fits well in both amplitude and phase for 4.5 wave cycles at altitudes below $-100 \mathrm{~km}$. However, the model does not reproduce the multiple amplitude peaks. The significant amplitude variation suggests beating wave modes whose wavelengths are unresolved by the data. The observed amplitude can be well modeled by superposing two sine waves that have vertical wavelengths $5.96 \mathrm{~km}$ and $6.66 \mathrm{~km}$ respectively.

For a given eddy diffusion coefficient, $K$, waves with short $\lambda_{z}$ and long $\lambda_{h}$ dissipate low in the atmosphere. This places a natural filtering mechanism for wavelengths that can propagate at a given altitude. In order for a wave with a short $\lambda_{z}$ (e.g., $6 \mathrm{~km}$ ) to propagate at the probed pressure levels, $\lambda_{h}$ must be rather small $(\sim 10 \mathrm{~km})$. The wave then comes close to violating the hydrostatic approximation $\left(\lambda_{z} \ll \lambda_{h}\right)$. It also raises a question about the detectability of such a wave by a stellar occultation, which averages the properties of the atmosphere along the line of sight. The length of this averaging on Saturn is about $\sqrt{2 \pi r H}=4500 \mathrm{~km}$, where $r$ is the planetary radius. Sicardy et al. (1999) demonstrate that the amplitude of light-curve fluctuations resulting from the presence of a monochromatic wave with a projection of the horizontal wavelength along the line of sight $l=\lambda_{h} / \cos \theta_{l}$ is not significantly reduced if

$$
\frac{l}{\lambda_{z}}>\left(\frac{r}{4 H}\right)^{0.5}=\eta
$$

where $0^{\circ}<\theta_{l}<90^{\circ}$ is the angle between the line of sight and the horizontal wave propagation direction. For Saturn, $\eta=18$. If the $6.5 \mathrm{~km}$ feature is to be interpreted as a gravity wave with a horizontal wavelength $\lambda_{h}=10 \mathrm{~km}$, the wave must be propagating at an angle $\theta_{l}>85^{\circ}$ in order to be detected during the occultation. In other words, we would have detected a wave that propagated almost along the planetary meridian. In this respect, of all the waves that might be present in the atmosphere, we preferentially detect waves that propagate in the horizontal at large angles $\theta_{l}$. In summary, the arguments for a wave interpretation of the very short scales $(5-8 \mathrm{~km})$ present in the temperature profile are easiest to accept if the wave field is isotropic.

To see how well the model-fitting method rejects noise, we performed the same analysis on one randomly selected profile from each noise set; results are in the final eight panels of Figure 4. For each type of noise realization we reconstruct two scales, one short (less than $30 \mathrm{~km}$ ) and one long (about 40-60 km) and fit gravity-wave models. The fits to reconstructions of noise and data show similar qualities. Short scales fit well over a few wavelengths but not through the entire altitude range, and large scales fit relatively well throughout the vertical range, but the region outside the COI contains less than two full wave cycles. This makes it difficult to identify an atmospheric wave based only on the goodness of fit. However, there are ways to distinguish some waves from noise. First, our detected waves have much higher amplitude than the noise at the same scale. The relevant scales appear significant in the global wavelet power spectrum (Figure 3), even though most span only a short vertical range. Second, in the noise fits most of the power (including the peak) is typically within the COI, and the amplitude varies with altitude faster than expected for a wave. Unfortunately, the latter are tendencies rather than robust 
Table 5

Parameters of the Modified Desaubies Fits

\begin{tabular}{|c|c|c|c|c|c|c|}
\hline Case & $a$ & $\begin{array}{c}m_{*} \\
\left(\mathrm{~km}^{-1}\right)\end{array}$ & $s$ & $t$ & $\begin{array}{l}L_{*}^{\mathrm{a}} \\
(\mathrm{km})\end{array}$ & $\chi^{2} / \mathrm{dof}^{\mathrm{b}}$ \\
\hline M Jupiter & 0.1000 & 0.207 & 0.00 & 3.00 & 30.3 & 780 \\
\hline B Jupiter & 0.1000 & 0.207 & 0.00 & 3.00 & 30.3 & 791 \\
\hline M nominal & 0.1000 & 0.273 & 0.00 & 3.00 & 23.0 & 720 \\
\hline B nominal & 0.1000 & 0.367 & 0.00 & 3.00 & 17.1 & 681 \\
\hline M fit 4 & $0.1098 \pm 0.0016$ & $0.564 \pm 0.043$ & $-1.71 \pm 0.09$ & $3.92 \pm 0.06$ & $11.1 \pm 0.9$ & 17 \\
\hline B fit 4 & $0.1089 \pm 0.0015$ & $0.725 \pm 0.056$ & $-1.95 \pm 0.07$ & $4.23 \pm 0.08$ & $8.7 \pm 0.7$ & 13 \\
\hline M fit 3 & $0.0848 \pm 0.0015$ & $0.299 \pm 0.006$ & 0.00 & $3.49 \pm 0.02$ & $21.0 \pm 0.4$ & 21 \\
\hline B fit 3 & $0.0872 \pm 0.0016$ & $0.313 \pm 0.006$ & 0.00 & $3.58 \pm 0.02$ & $20.1 \pm 0.4$ & 19 \\
\hline
\end{tabular}

Notes.

${ }^{\mathrm{a}} L_{*}=2 \pi / m_{*}$.

b dof: degrees of freedom. Note that we fit only the broadband spectrum, not the waves.

discriminators; real waves can do the same, making it difficult to specify robust criteria to discriminate between waves and noise-induced features. For example the fits of the $40 \mathrm{~km}$ data structure (D40) and the $40 \mathrm{~km}$ noise structure (GN40) look very similar. The amplitude of the fit to the real data is, however, more than five times larger than the fit to the noise. This signal-tonoise assessment is thus the only reliable discriminator known to us.

We conclude that, while this method may do well at quantifying the properties of strong waves, it is not a good discriminator between weak waves and noise. A number of criteria should be satisfied for the entire region outside the COI to make a positive wave identification.

1. The wave amplitude should be many times the mean amplitude of the worst-case real noise at that $\lambda_{z}$.

2. A wave model should fit both the amplitude and phase of the observed temperature fluctuations well.

3 . The structure should have more than a few cycles.

Note that structures failing one or more criteria may still be (or contain) waves. We discuss the broad spectrum of weaker gravity waves in the next section.

\section{POWER SPECTRUM}

To compare the power spectrum of temperature fluctuations in Saturn's atmosphere with that in other atmospheres, we calculate global spectra for normalized thermal profiles, $(T-\bar{T}) / \bar{T}$, where $\bar{T}$ is the mean temperature. While others (e.g., Young et al. 2005; Allen \& Vincent 1995) have simply chosen regions of their data where the background was clearly isothermal to calculate $\bar{T}$, we do not have this luxury, so we computed it in two ways. In the first, $\bar{T}$ is the mean temperature $(148.04 \mathrm{~K})$ in the good region of the profile. In the second, $\bar{T}$ is the wavelet-reconstructed thermal profile for $\lambda_{z}>60 \mathrm{~km}$ (the smooth, solid line in the left panel of Figure 2). The choice of $60 \mathrm{~km}$ is arbitrary, hence the two cases. We call them $\mathrm{M}$ and $\mathrm{B}$, for mean and background, respectively, and present them in Figure 5.

From their wavelet transforms, we derived the global power spectrum for both normalized temperatures from our nominal profile (75\% cap), as described in Section 5. To derive noise spectra, we calculated the wavelet spectrum of each noise inversion, then found the mean power at each wavelength over all the altitudes and inversions in each set, excluding points in the COI. Since we demonstrated above (Figure 4) that real noise can induce wave-like features, we also derived spectra based on the maximum rather than the mean, to ensure that anything



Figure 5. Normalized temperature profiles derived from the $75 \%$ cap inversion. The thick profile uses the wavelet-smoothed background profile of Figure 2 (B case). The thin profile uses the mean of the temperature profile in the altitude range presented ( $\mathrm{M}$ case).

we call real must stand well above these spurious features. Figures 6 and 7 and Table 3 present the resulting power spectral densities and power-law fits, computed with the full, waveletnormalized power spectrum for comparison to other work. The table also gives power-law fits to temperature amplitude, with neither wavelet nor temperature normalizations applied.

The noise sets' power spectra follow an $m^{-3}$ dependence, where $m$ is the wavenumber that is very consistent along their entire lengths, save for small tails near the Nyquist frequency. The tails are well below the resolution of the uninterpolated light curve, so we exclude them from the fits. The non-white noise should be due almost entirely to Earth's atmosphere. The $m^{-3}$ power law is unfortunate, as several atmospheric processes share it. These include the short-scale end of the universal gravitywave spectrum observed on Earth and Jupiter (see below) and short-scale, 2D turbulence (Harrington et al. 1996; Travis 1978), although 2D dynamics, at least, likely break down at scales larger than are relevant to the current observations. The scaled 


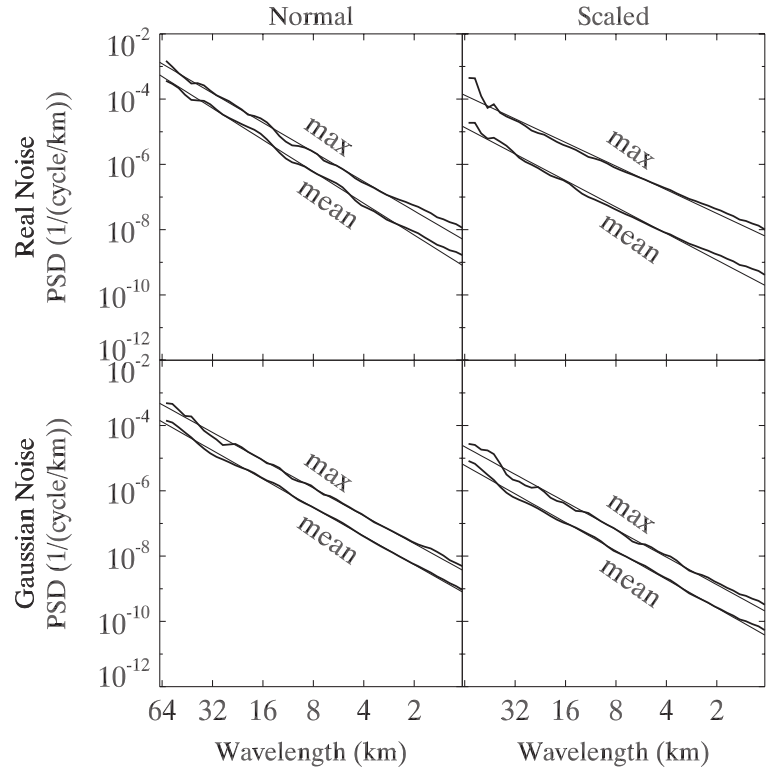

Figure 6. Power spectra of inverted noise-test light curves (thick lines), and power-law fits (thin lines). The words "max" and "mean" indicate global spectra generated by those two methods from each of the four types of noise tested (see the text). Parameters of these fits appear in Table 3. We compare them to the data in Figure 7.

noise sets had lower power (see Figure 6 and constants in Table 3), but otherwise behaved similarly to their unscaled brethren. The spectra computed by taking the maximum lay a factor of 4 or more above those computed by taking the mean, which the fit constants of Table 3 reflect.

Figure 7 presents the data and fits to the noise spectra (and spectrum models; see below). At all wavelengths, the power spectrum of the data is $2-10$ times higher than even the most conservative noise spectrum (labeled "RNX") and departs from the very linear shape of the noise spectra. We can confidently accept that the data's spectrum reflects properties of Saturn's atmosphere rather than noise. The log plot allows by-eye $\mathrm{S} / \mathrm{N}$ calculation under different noise models. For example, at a wavelength of $32 \mathrm{~km}$, the data PSD is $10^{-3}$ and the RNX noise spectrum is $2 \times 10^{-4}$, indicating a $5 \sigma$ detection of the planetary spectrum there. Under GNX noise it would be $10 \sigma$, and under GNM noise it would be a $50 \sigma$ detection. We thus conclude that comparison to real, not Gaussian, noise is necessary and that averaging over the maximum power at each wavelength and location in the noise set (rather than the mean) is necessary because of the spurious, wave-like features induced by real noise.

We can now investigate the spectrum of gravity waves on Saturn, though with the caveat that temperature profiles derived from occultations give an average over a long horizontal path through the atmosphere (see above). On Earth, the spectrum of temperature fluctuations is constant enough over season and location to be called the "universal spectrum" (Van Zandt 1982; Balsley \& Carter 1982; Dewan et al. 1984; Vincent 1984; Smith et al. 1987; Tsuda et al. 1989). It has been observed on Jupiter (Young et al. 2005) as well, and follows the modified Desaubies function,

$$
p(m)=a \frac{N^{4}}{g^{2} m_{*}^{3}} \frac{\left(m / m_{*}\right)^{s}}{1+\left(m / m_{*}\right)^{s+t}},
$$

where $p$ is the PSD, $a$ is a unitless constant, $N$ is the Brunt-Väisälä frequency, $m_{*}=|\Gamma| / 2 \sigma_{T}=2 \pi / L_{*}$ is the critical wavenumber, $L_{*}$ is the critical wavelength, $\sigma_{T}$ is the rms temperature fluctuation of $(T-\bar{T}), s$ is the long-wavelength power-law exponent, and $t$ is the short-wavelength exponent.

The characteristic wavenumber $m_{*}$ divides the spectrum into two regions: a small wavenumber region $\left(m<m_{*}\right)$ and a large wavenumber region $\left(m>m_{*}\right)$. The shape and magnitude in


Figure 7. Global power spectrum of the data (thick line) compared to data fits (smoothly curving lines, see key) and noise spectrum fits (straight lines). This compact presentation relates the main points of this paper; see Section 7 for interpretation. Mean-normalized data and fits are on the left, background-normalized on the right. The noise spectra fits are error estimates for the data spectrum under our eight noise models for power spectra of inversions. Table 3 gives parameters of these fits. The three characters of each noise label indicate, in order, the type of noise set (Real or Gaussian), whether it is a Normal or Scaled set, and whether the spectrum came from a vertical average over the maXima or Means of the power at each location in the wavelet spectra of the inversions in that noise set. For example, "RNX" means Real noise, Normal (non-scaled), averaged vertically over the maXima. Mean spectra have dashed lines; maximum spectra have dotted lines. Standing many times higher than the linear noise spectra, the data show peaks superposed on the modified Desaubies function (Equation (2)) that describes the "universal spectrum" of gravity waves for Earth. The four smoothly curving traces are the modified Desaubies fits given in the key and Table 5. The peaks are the discrete waves shown in Figure 4, analyzed in Section 6, and presented in Table 4. They cause the large reduced- $\chi^{2}$ values of Table 5. 
the small-wavenumber region (large vertical wavelengths) is believed to be dominated by the wave source characteristics and is proportional to $m^{s}$. The exponent $s$ for the terrestrial atmosphere is not well constrained, though it is typically about 1 . In the large-wavenumber regime (small wavelengths) saturation and/or dissipation processes are believed to control the wave spectrum (Gardner 1996 and references therein). In this region the spectrum is proportional to $m^{-t}$, where $t=3$. The overall amplitude of the spectrum is controlled by the dimensionless parameter $a$, which may represent the wave generation mechanism.

Young et al. (2005) applied terrestrial parameters $a=0.1, s=$ 0 , and $t=3$, and derived $N=0.0176 \mathrm{~s}^{-1}, \Gamma=2.11 \mathrm{~K} \mathrm{~km}^{-1}$, and $\sigma_{T}=5.0 \mathrm{~K}$ to compute $L_{*}=30.3 \mathrm{~km}$ and thus the Desaubies function for Jupiter. Note that in their paper, a typographical error gives $m_{*}=\Gamma \sigma_{T} / 2$, but the value is calculated correctly. Their Equation (3) gives a curve that lies a factor of $\sim 2 \pi$ lower than the curve presented in their Figure 7, using the parameters given above, though the shape is the same. It is unclear whether the plotted data have been similarly shifted or whether the two traces do not in fact follow one another.

Figure 7 presents Equation (2) evaluated for the parameters given in Table 5. Some of these curves have free parameters fit with a Levenberg-Marquardt minimizer; the uncertainties on those parameters are from the minimizer's covariance matrix. The "Jupiter" case in Table 5 and Figure 7 uses $m_{*}$ from Young et al. (2005), but $N=0.01165 \mathrm{~s}^{-1}$ and $\Gamma=0.992 \mathrm{~K} \mathrm{~km}^{-1}$, appropriate to our data. The poor visual fit and high $\chi^{2}$ show that it misses the data by a substantial margin. Using $\sigma_{T}$ calculated from the $\mathrm{B}$ and $\mathrm{M}$ cases (1.18 and $1.98 \mathrm{~K}$, respectively) yields the $m_{*}$ and $L_{*}$ given in the "nominal" cases (i.e., Saturnian values and no free parameters). Things are better, but not good. However, the long atmospheric path of the occultation ray likely reduced $\sigma_{T}$ well below what would be observed in situ; for the Galileo Probe data, $\sigma_{T}=5.0 \mathrm{~K}$, for example. For the case where all parameters vary, $a$ is still comfortably close to 0.1 . Fixing $s=0$ puts $a$ closer to 0.085 . In all cases with free exponents, the exponents move substantially away from their nominal values. In particular, $t$ is now decidedly steeper than the -3 power law usually cited for gravity wave spectra. Since waves with small $\lambda_{z}$ are more likely to have small $\lambda_{h}$, the steeper power law may be due to preferential filtering of short wavelengths due to averaging along the occultation ray path.

The reduced $\chi^{2}$ is still high, indicating that the undulations in the spectrum are significant: there is more here than a background spectrum of gravity waves. The gravity-wave model presented above fits averages over the sections of the wavelet transform that produce these bumps. That model assumes a single wavelength per wave, so we cannot use its output to make a complete, simultaneous model of background and discrete waves that might fit the global spectrum more perfectly.

\section{DISCUSSION OF THE SPECTRUM}

The one-dimensional power spectrum of terrestrial gravity waves exhibits a nearly universal behavior in its large wavenumber region (see the previous section). This statement is based on a large number of observations of the vertical structure of the horizontal wind, temperature, and density fluctuations of the present wave modes using diverse experimental techniques. The shape of the spectrum at large vertical wavenumbers (short vertical wavelengths) is consistently independent of time, place, and altitude. This is usually attributed to a saturation process that limits the wave amplitude growth.
Theoretical work diverges on the nature of the saturation process at work. The existing theories use different physical mechanisms for dissipating wave energy including shear and convective instabilities (Dewan \& Good 1986), cascade processes (Dewan 1991), wave-induced Doppler effects (Hines 1991), and wave-induced diffusion (Gardner 1994; Zhu 1994). The different theories all predict the same shape and behavior for the PSD, including the -3 slope of the large wavenumber tail and the presence of a characteristic wavenumber $m_{*}$ that limits the range of the saturated spectrum. Since $m_{*} \propto 1 / \sigma_{T}$, the theories predict a decrease in $m_{*}$ with altitude as $m_{*} \propto e^{-1 / 4 H}$, and the terrestrial data agree.

Despite the diversity of wave-generating mechanisms and atmospheric thermal structure on different planets, the saturation theories are based on rather general concepts, implying similar spectra on different planets. Confirming this on other planets is challenging as it requires local measurements at a variety of altitudes and horizontal locations on each planet, yet there have been only a few atmospheric entry probes. Occultations average over a long path length, underestimating the wave amplitudes. This effect depends on wavelength and probably modifies the true spectral slope, especially at large wavenumbers. Indeed, in Earth's atmosphere a typical value for $t$ is 2.5-3 (Allen \& Vincent 1995), whereas our best fits show a slope of 3.5-4.2. The higher values for $t$ might also be real, reflecting actual differences between the dominant saturation processes acting in Earth's and Saturn's atmospheres. Gravity wave studies in Earth's atmosphere are based on thousands of temperature profiles taken at different locations, altitudes, and times of the year, using ground-based, airborne, and satellite-based techniques, whereas we are discussing a single temperature profile. On the other hand, the value of $L_{*} \approx 15-25 \mathrm{~km}$, based on the observed temperature variance (see Equation (2)), is in good agreement with the value determined using the Desaubies fits (see three-parameter fits in Table 5).

Where should we focus our efforts for wave detection? In addition to seeking individual wave modes that may reveal information about atmospheric structure before losing their identity through interactions, one can look at the spectral characteristics at small wavenumbers (large vertical wavelengths). The theory says that at wavenumbers smaller than $m_{*}$, the waves are not affected by the saturation processes (whatever they are) and retain the spectral characteristics of the generation mechanism. Numerical simulations of convectively generated gravity waves show that there is also a dependence between the characteristic vertical wavenumber $m_{*}$ and the depth of the convective cell generating the waves (Fritts \& Alexander 2003).

\section{CONCLUSIONS}

We have analyzed a light curve based on IRTF observations of the 1998 November 14 occultation of GSC 0622-00345 by Saturn (Paper I). We presented and analyzed isothermal lightcurve fits, an atmospheric thermal profile, a wavelet analysis, gravity wave modeling, and power spectra. The derived thermal profile varies over $142-151 \mathrm{~K}$ in the pressure range $1-60 \mu \mathrm{bar}$ at a latitude of $55.5 \mathrm{~S}$. The vertical temperature gradient is removed by more than $0.2 \mathrm{~K} \mathrm{~km}^{-1}$ from the adiabatic lapse rate, indicating that the stratospheric region sounded by the occultation is statically stable. Our thermal gradient profile shows the same alternating-rounded-spiked appearance of other occultation profiles, including one for Saturn (Cooray et al. 1998). This shape has previously been interpreted as evidence of gravity wave breaking (Raynaud et al. 2003, 2004; Young et al. 2005). 
Our new noise tests, based on real noise sampled from our light curve's upper baseline rather than synthetic, uncorrelated Gaussian noise, showed that atmospheric scintillation (and similar correlated noise sources such as spacecraft pointing drifts) can introduce relatively strong, spurious temperature fluctuations into the thermal profile derived by inverting an atmospheric occultation light curve. For our data, the effect was many $\mathrm{K}$ for the raw inversions, but it mainly appeared at longer wavelengths in power spectra. However, the amplitudes at the shorter wavelengths found outside the COI were still of order $0.1-1 \mathrm{~K}$, comparable to the amplitudes of the gravity waves often identified in occultation data sets. We thus developed several significance tests for our power-spectrum analyses, and note that without such tests, one must be skeptical of gravity-wave detection claims in ground-based (and possibly some spacebased) occultation inversions.

We used a wavelet analysis to search for localized gravity wave trains. Based on the wavelet power and the use of significance tests only, the strongest candidate had an amplitude of $0.7 \mathrm{~K}, \lambda_{z}=12.5 \mathrm{~km}$ at $z_{\max }=-35 \mathrm{~km}$, and lasted four cycles. It and several shorter-wavelength features stand over the global power spectrum at the $95 \%$ confidence level or greater. Alternative explanations for the observed periodic structures include sound waves, planetary waves, and nontransient features. Without knowing the horizontal structure of the wave we cannot rule out sound waves or planetary waves as the cause for the temperature fluctuations. However, one can make the argument that, for a given $\lambda_{z}$, planetary waves typically have lower (time) frequencies and therefore dissipate lower in the atmosphere than the valid region of our profile.

To take into account both phase and amplitude information, which could improve sensitivity over our amplitude-based noise limit, we fit a gravity-wave model to the amplitude and phase of the strongest features in the valid region of the wavelet spectrum, following Raynaud et al. (2004). The model calculates the temperature amplitude versus height of a single, damped wave mode propagating throughout our profile's valid region. We performed fits both with and without a new parameter for constant vertical wind shear. The added parameter did not improve the fits, so we report the shearless fits here for consistency with prior results from this model.

Our best candidate for a gravity wave that propagates continuously through the valid region, as assessed by our model, had $\lambda_{z}=40 \mathrm{~km}, \lambda_{h}=200 \mathrm{~km}$, and a period of 42 minutes. At this altitude the wave is not strongly affected by dissipation and achieves a maximum amplitude above the observed atmospheric region exceeding $1 \mathrm{~K}$. According to Equation (1), this wave was detectable if the angle between the line of sight and the horizontal direction of wave propagation exceeded $74^{\circ}$. However, similar fits to quasi-periodic features in thermal profiles derived from isothermal (i.e., waveless) light curves with real noise gave some fits of similar appearance. This demonstrates the need for a study of real noise to establish an amplitude criterion that discriminates real waves from noise. The reason wavelike (i.e., sinusoidal) features arise out of the noise is simple: by reconstructing only a limited range of wavelengths, the resulting profile is certainly sinusoidal and has a favored period. Wave models are sinusoidal, and ours even has parameters that allow the phase and amplitude to vary, so we will get a good fit if the amplitude of the reconstructed data does not vary much. We find that this circumstance occurs in the noise data sets often enough to require at least criteria for significant wave amplitude. Criteria involving the number of cycles outside the COI or the phase of the reconstructed data could potentially provide even-more-stringent limits.

We derived global power spectra from our wavelet transforms, using only data outside the COI. This method greatly reduced the noise level of the spectra, which stand everywhere 2-10 times above the noise level calculated using real-noise-contaminated isothermal light curves. The power spectra follow the modifiedDesaubies form of the universal spectrum of gravity waves, though with a slightly more negative high-wavenumber exponent. Superposed on this spectrum one sees the signature of the discrete wave structures discussed above. That we see both the universal spectrum and individual features fit well by gravitywave models lends confidence that we are indeed looking at a signal dominated by gravity waves.

The amplitudes of all five wave-like features that we analyzed are well above the noise level. The wave model used to fit these features is based on the assumption that the waves propagate independently of each other. Interactions between the wave modes might be able to explain the discrepancies between the observed wave fluctuations and the model's single gravity waves. Note that the discrepancies are more significant when derived wavenumbers are larger than the observationally derived characteristic wavenumber. This is the saturated part of the spectrum where wave-wave interactions determine wave behavior and vertical propagation.

Direct comparison of our derived temperatures with previous Saturn occultation measurements (see, e.g., Table VII of Hubbard et al. 1997) requires care, both because of differences in the assumed mean molecular mass and because Saturn's mean stratospheric temperature is strongly affected by seasonally varying insolation (Bézard \& Gautier 1985). Heating by inertia-gravity waves might also be important, at least in some regions and/or seasons (Cooray et al. 1998; French \& Gierasch 1974; Young et al. 1997). Detailed modeling of stratospheric temperatures requires taking account of non-LTE effects as well (Appleby 1990). A clearer picture of zonal and seasonal variations

in

Saturn's stratospheric structure should emerge when Earthbased stellar occultations can be viewed in the context of data from the Cassini orbiter.

We thank L. A. Young for helpful discussions, and reviewers J. L. Elliot and E. Lellouch for comments that significantly improved this paper. We thank the NASA Astrophysics Data System, JPL Solar System Dynamics group, and the free and opensource software communities for software and services. Free wavelet software (http://paos.colorado.edu/research/wavelets/) was provided by C. Torrence and G. Compo. This investigation was supported by Wellesley College under NASA Contract 961169 and by the NASA PGG program.

Facilities: IRTF

\section{REFERENCES}

Allen, S. J., \& Vincent, R. A. 1995, J. Geophys. Res., 100, 1327

Appleby, J. F. 1990, Icarus, 85, 355

Atreya, S. K. 1986, Atmospheres and Ionospheres of the Outer Planets and their Satellites (Berlin: Springer)

Balsley, B. B., \& Carter, D. A. 1982, Geophys. Res. Lett., 9, 465

Bézard, B., \& Gautier, D. 1985, Icarus, 61, 296

Bosh, A. S., \& McDonald, S. W. 1992, AJ, 103, 983

Brown, R. H., et al. 2004, Space Sci. Rev., 115, 111

Conrath, B. J., \& Gautier, D. 2000, Icarus, 144, 124

Cooray, A. R., \& Elliot, J. L. 2003, ApJ, 587, L121

Cooray, A. R., Elliot, J. L., Bosh, A. S., Young, L. A., \& Shure, M. A. 1998, Icarus, 132, 298 
Creasey, J. E., Forbes, J. M., \& Hinson, D. P. 2006, Geophys. Res. Lett., 33 L01803

Dewan, E. M. 1991, Geophys. Res. Lett., 18, 1473

Dewan, E. M., \& Good, R. E. 1986, J. Geophys. Res., 91, 2742

Dewan, E. M., Grossbard, N., Quesada, A., \& Good, R. E. 1984, Geophys. Res. Lett., 11, 80

Elliot, J. L., Person, M. J., \& Qu, S. 2003, AJ, 126, 1041

Elliot, J. L., \& Young, L. A. 1992, AJ, 103, 991

Flasar, F. M., et al. 2005, Science, 307, 1247

Fletcher, L. N., Orton, G. S., Teanby, N. A., \& Irwin, P. G. J. 2009, Icarus, 202, 543

Fouchet, T., Guerlet, S., Strobel, D. F., Simon-Miller, A. A., Bézard, B., \& Flasar, F. M. 2008, Nature, 453, 200

French, R. G., Elliot, J. L., \& Gierasch, P. J. 1978, Icarus, 33, 186

French, R. G., \& Gierasch, P. J. 1974, J. Atmos. Sci., 31, 1707

French, R. G., Nicholson, P. D., \& Matthews, K. 1999, in Proc. 5th Workshop on Observations of Mutual Events PHEMU97, ed. J. Arlot \& C. Blanco (Paris: Institut de Méchanique Céleste, Bureau des Longitudes), 163

Fritts, D. C., \& Alexander, M. J. 2003, Rev. Geophys., 41, 1003

Fritts, D. C., Wang, L., \& Tolson, R. H. 2006, J. Geophys. Res., 111, A12304

Gardner, C. S. 1994, J. Geophys. Res., 99, 20601

Gardner, C. S. 1996, J. Atmos. Terr. Phys., 58, 1575

Harrington, J., Dowling, T. E., \& Baron, R. L. 1996, Icarus, 124, 32

Harrington, J., \& French, R. G. 2010, ApJ, 716, 398 (Paper I)

Hines, C. O. 1991, J. Atmos. Sci., 48, 1361

Hinson, D. P., \& Jenkins, J. M. 1995, Icarus, 114, 310

Hubbard, W. B., McCarthy, D. W., Kulesa, C. A., Benecchi, S. D., Person, M. J., Elliot, J. L., \& Gulbis, A. A. S. 2009, Icarus, 204, 284

Hubbard, W. B., et al. 1997, Icarus, 130, 404

Kliore, A. J., Lindal, G. F., Patel, I. R., Sweetnam, D. N., Hotz, H. B., \& McDonough, T. 1980, Science, 207, 446

Kliore, A. J., Nagy, A. F., Marouf, E. A., Anabtawi, A., Barbinis, E., Fleischman, D. U., \& Kahan, D. S. 2009, J. Geophys. Res., 114, A04315

Lindal, G. F., Sweetnam, D. N., \& Eshleman, V. R. 1985, AJ, 90, 1136

Lindzen, R. S. 1981, J. Geophys. Res., 86, 9707
Matcheva, K. I., \& Strobel, D. F. 1999, Icarus, 140, 328

Moses, J. I., Bézard, B., Lellouch, E., Gladstone, G. R., Feuchtgruber, H., \& Allen, M. 2000, Icarus, 143, 244

Nagy, A. F., et al. 2006, J. Geophys. Res., 111, A06310

Nicholson, P. D., McGhee, C. A., \& French, R. G. 1995, Icarus, 113, 57

Person, M. J., et al. 2008, AJ, 136, 1510

Raynaud, E., Matcheva, K., Drossart, P., Roques, F., \& Sicardy, B. 2004, Icarus, 168,324

Raynaud, E., et al. 2003, Icarus, 162, 344

Roques, F., et al. 1994, A\&A, 288, 985

Shemansky, D. 2008, in Proc. 37th COSPAR Scientific Assembly, Symp. C, Session 32, 2840

Sicardy, B., et al. 1999, Icarus, 142, 357

Smith, G. R., Shemansky, D. E., Holberg, J. B., Broadfoot, A. L., Sandel, B. R., \& McConnell, J. C. 1983, J. Geophys. Res., 88, 8667

Smith, S. A., Fritts, D. C., \& Van Zandt, T. E. 1987, J. Atmos. Sci., 44, 1404

Toigo, A., Gierasch, P., Sicardy, B., \& Lellouch, E. 2010, Icarus, in press

Torrence, C., \& Compo, G. P. 1998, Bull. Am. Meteorol. Soc., 79, 61

Travis, L. D. 1978, J. Atmos. Sci., 35, 1584

Tsuda, T., Inoue, T., Kato, S., Fukao, S., Fritts, D. C., \& Vanzandt, T. E. 1989, J. Atmos. Sci., 46, 2440

Tyler, G. L., Eshleman, V. R., Anderson, J. D., Levy, G. S., Lindal, G. F., Wood, G. E., \& Croft, T. A. 1981, Science, 212, 201

Tyler, G. L., Eshleman, V. R., Anderson, J. D., Levy, G. S., Lindal, G. F., Wood G. E., \& Croft, T. A. 1982, Science, 215, 553

Van Zandt, T. E. 1982, Geophys. Res. Lett., 9, 575

Vincent, R. A. 1984, J. Atmos. Terr. Phys., 44, 119

Young, E. F., et al. 2008, AJ, 136, 1757

Young, L. A., Bosh, A. S., Buie, M., Elliot, J. L., \& Wasserman, L. H. 2001, Icarus, 153, 236

Young, L. A., Yelle, R. V., Young, R., Seiff, A., \& Kirk, D. B. 1997, Science, 276, 108

Young, L. A., Yelle, R. V., Young, R., Seiff, A., \& Kirk, D. B. 2005, Icarus, 173, 185

Zhu, X. 1994, J. Atmos. Sci., 51, 3615 